\title{
Tandem Aldol-Allylation and Aldol-Aldol Reactions with Ketone-Derived Enolsilanes: Highly Diastereoselective Single Step Synthesis of Complex Tertiary Carbinols
}

\author{
Xiaolun Wang, Qinglin Meng, Nicholas R. Perl, Yue Xu, and James L. Leighton* \\ Department of Chemistry, Columbia University, New York, New York, 10027
}

\section{Supporting Information}

General Information. All reactions were carried out under an atmosphere of nitrogen in flame- or oven-dried glassware with magnetic stirring unless otherwise indicated. Degassed solvents were purified by passage through an activated alumina column. Allyltrichlorosilane, cis-crotyltrichlorosilane, transcrotyltrichlorosilane, methallyltrichlorosilane, and (Z)-2-methylbut-2-enyltrichlorosilane were prepared according to published procedures. ${ }^{1}{ }^{1} \mathrm{H}$ NMR spectra were recorded on a Bruker DMX-500 (500 MHz) spectrometer and are reported in ppm from $\mathrm{C}_{6} \mathrm{D}_{6}$ internal standard $(7.15 \mathrm{ppm})$. Data are reported as follows: $(\mathrm{s}=$ singlet, br $\mathrm{s}=$ broad singlet, $\mathrm{d}=$ doublet, $\mathrm{t}=$ triplet, $\mathrm{q}=$ quartet, quin = quintet, $\mathrm{sep}=$ septet, $\mathrm{m}=$ multiplet, $\mathrm{dd}=$ doublet of doublets, $\mathrm{td}=$ triplet of doublets, $\mathrm{tt}=$ triplet of triplets, $\mathrm{dq}=$ doublet of quartets, $\mathrm{ddt}=$ doublet of doublet of triplets; coupling constant(s) in Hz; integration; assignment). Proton decoupled ${ }^{13} \mathrm{C}$ NMR spectra were recorded on a Bruker DPX-400 (100 MHz) and are reported in ppm from $\mathrm{C}_{6} \mathrm{D}_{6}$ internal standard (128.0 ppm). Infrared spectra were recorded on a Perkin Elmer Paragon 1000 FT-IR spectrometer.

(1) (a) Tsuji, J.; Hara, M.; Ohno, K. Tetrahedron 1974, 30, 2143-2146. (b) Kira, M.; Hino, T.; Sakurai, H. Tetrahedron Lett. 1989, 30, 1099-1102. (c) Iseki, K.; Kuroki, Y.; Takahashi, M.; Kishimoto, S.; Kobayashi, Y. Tetrahedron 1997, 53, 3513-3526. (d) Furuya, N.; Sukawa, T. J. Organomet. Chem. 1975, 96, C1-C3. (e) Fleming, I.; Lee, D. J. Chem. Soc. Perkin Trans. 1 1998, 2701-2709. 
Page S2

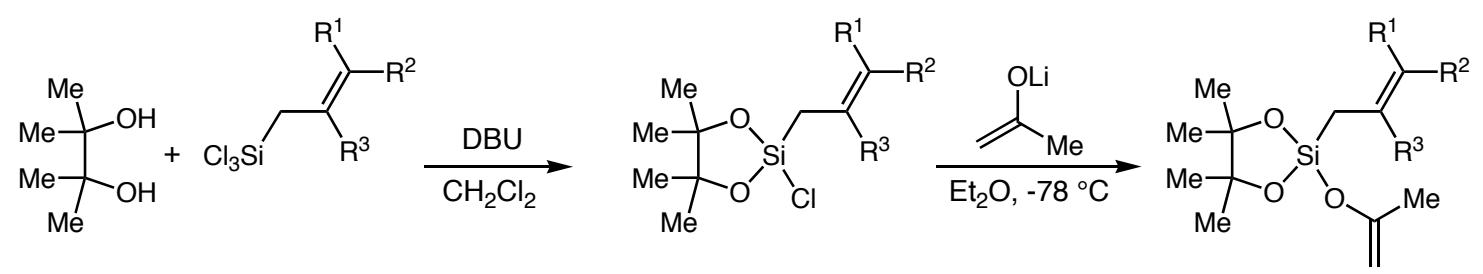

\section{General procedure for the preparation of allylenolsilanes 1, 4, 6, 8, and 10:}

To a cooled $\left(0{ }^{\circ} \mathrm{C}\right)$ solution of the requisite allyltrichlorosilane $(34 \mathrm{mmol})$ in $\mathrm{CH}_{2} \mathrm{Cl}_{2}(50 \mathrm{~mL})$ was added 1,8-Diazabicyclo[5.4.0]undec-7-ene $(84 \mathrm{mmol})$. A solution of pinacol $(34 \mathrm{mmol})$ in $\mathrm{CH}_{2} \mathrm{Cl}_{2}(50$ $\mathrm{mL}$ ) was then added SLOWLY and the resultant solution was warmed to room temperature and stirred for 12 hours. The solution was concentrated and the residue was treated with diethylether $(100 \mathrm{~mL})$. The mixture was stirred for 1 hour during which time the formation of a precipitate was observed. The mixture was then filtered and the filtrate was concentrated. The residue was distilled in vacuo to give the allylchlorosilanes as clear, colorless liquids (50-70\% yield).

To a cooled $\left(0{ }^{\circ} \mathrm{C}\right)$ solution of $\mathrm{MeLi}\left(20 \mathrm{mmol}, 1.6 \mathrm{M}\right.$ in $\left.\mathrm{Et}_{2} \mathrm{O}\right)$ was added 2(trimethylsilyloxy)propene $(20 \mathrm{mmol})$. After $30 \mathrm{~min}$ the solution was warmed to room temperature and was stirred for 1 hour during which time the formation of a white precipitate was observed. The mixture was cooled to $-78{ }^{\circ} \mathrm{C}$, and the allylchlorosilane $(20 \mathrm{mmol})$ was added. The solution was warmed to room temperature and stirred for 5 hours. The solution was diluted with pentane $(20 \mathrm{~mL})$, and the mixture was then filtered through a pad of celite. The filtrate was concentrated, and the residue was distilled in vacuo to give the allylenolsilanes as clear, colorless liquids (60-70\% yield).

Allylenolsilane 1: ${ }^{1} \mathrm{H}$ NMR $\left(400 \mathrm{MHz}, \mathrm{C}_{6} \mathrm{D}_{6}\right) \delta$ 5.82-5.93 (m, 1H, $\left.\mathrm{CH}_{2}=\mathrm{CH}\right)$, 5.01-5.08 (m, 1H, one of $\left.\mathrm{CH}_{2}=\mathrm{CH}\right), 4.94-4.98\left(\mathrm{~m}, 1 \mathrm{H}\right.$, one of $\left.\mathrm{CH}_{2}=\mathrm{CH}\right), 4.57\left(\mathrm{~s}, 1 \mathrm{H}\right.$, one of $\left.\mathrm{CH}_{2}=\mathrm{CCH}_{3}\right), 4.15(\mathrm{~s}, 1 \mathrm{H}$, one of $\left.\mathrm{CH}_{2}=\mathrm{CCH}_{3}\right), 1.83\left(\mathrm{~d}, 2 \mathrm{H}, J=7.8 \mathrm{~Hz}, \mathrm{CH}_{2} \mathrm{Si}\right), 1.74\left(\mathrm{~s}, 3 \mathrm{H}, \mathrm{CH}_{2}=\mathrm{CCH}_{3}\right), 1.19 \quad(\mathrm{~s}, 6 \mathrm{H}$, six of $\left.\left(\mathrm{CH}_{3}\right)_{2} \mathrm{CC}\left(\mathrm{CH}_{3}\right)_{2}\right), 1.10$ (s, 6H, six of $\left.\left(\mathrm{CH}_{3}\right)_{2} \mathrm{CC}\left(\mathrm{CH}_{3}\right)_{2}\right) ;{ }^{13} \mathrm{C} \mathrm{NMR}\left(100 \mathrm{MHz}, \mathrm{C}_{6} \mathrm{D}_{6}\right) \delta 154.4,131.9,115.8$, 94.0, 81.7, 25.8, 25.7, 22.1, 19.0; ${ }^{29} \mathrm{Si}$ NMR (59 MHz, $\mathrm{CDCl}_{3}$ ) $\delta$-33.9; IR (film) 3114, 3081, 2980, 2927 , $1644,1445,1390,1372,1270,1145,1064,1008,971,933,902,881,836,815,782,695 \mathrm{~cm}^{-1}$.

Crotylenolsilane 4: ${ }^{1} \mathrm{H}$ NMR $\left(400 \mathrm{MHz}, \mathrm{C}_{6} \mathrm{D}_{6}\right) \delta$ 5.55-5.64 $\left(\mathrm{m}, 1 \mathrm{H}, \mathrm{CH}_{3} \mathrm{CH}=\mathrm{CH}\right)$, 5.42-5.51 (m, $1 \mathrm{H}$, $\left.\mathrm{CH}_{3} \mathrm{CH}=\mathrm{CH}\right), 4.58\left(\mathrm{~s}, 1 \mathrm{H}\right.$, one of $\left.\mathrm{CH}_{2}=\mathrm{CCH}_{3}\right), 4.15\left(\mathrm{~m}, 1 \mathrm{H}\right.$, one of $\left.\mathrm{CH}_{2}=\mathrm{CCH}_{3}\right), 1.83(\mathrm{dm}, 2 \mathrm{H}, J=8.3$ 
$\left.\mathrm{Hz}, \mathrm{CH}_{2} \mathrm{Si}\right), 1.75\left(\mathrm{~d}, 3 \mathrm{H}, J=0.6 \mathrm{~Hz}, \mathrm{CH}_{2}=\mathrm{CCH}_{3}\right), 1.57\left(\mathrm{dm}, 3 \mathrm{H}, J=6.6 \mathrm{~Hz}, \mathrm{CH}_{3} \mathrm{CH}=\mathrm{CH}\right), 1.20(\mathrm{~s}, 6 \mathrm{H}$, six of $\left.\left(\mathrm{CH}_{3}\right)_{2} \mathrm{CC}\left(\mathrm{CH}_{3}\right)_{2}\right), 1.12\left(\mathrm{~s}, 6 \mathrm{H}\right.$, six of $\left.\left(\mathrm{CH}_{3}\right)_{2} \mathrm{CC}\left(\mathrm{CH}_{3}\right)_{2}\right) ;{ }^{13} \mathrm{C} \mathrm{NMR}\left(100 \mathrm{MHz}, \mathrm{CDCl}_{3}\right) \delta 153.6$, 124.2, 122.2, 93.6, 81.6, 25.8, 25.7, 22.2,12.8, 12.4; ${ }^{29} \mathrm{Si} \mathrm{NMR} \mathrm{(59} \mathrm{MHz,} \mathrm{CDCl}_{3}$ ) $\delta$-33.6; IR (film) 3022, 2980, 2927, 1648, 1445, 1390, 1370, 1271, 1146, 1064, 1008, 972, 933, 880, 818, 697, 670, $639 \mathrm{~cm}^{-1}$.

Crotylenolsilane 6: ${ }^{1} \mathrm{H}$ NMR $\left(400 \mathrm{MHz}, \mathrm{C}_{6} \mathrm{D}_{6}\right) \delta$ 5.47-5.57 (m, 1H, $\left.\mathrm{CH}_{3} \mathrm{CH}=\mathrm{CH}\right)$, 5.36-5.46 (m, 1H, $\left.\mathrm{CH}_{3} \mathrm{CH}=\mathrm{CH}\right), 4.59\left(\mathrm{~s}, 1 \mathrm{H}\right.$, one of $\left.\mathrm{CH}_{2}=\mathrm{CCH}_{3}\right), 4.16\left(\mathrm{~s}, 1 \mathrm{H}\right.$, one of $\left.\mathrm{CH}_{2}=\mathrm{CCH}_{3}\right), 1.80(\mathrm{dq}, 2 \mathrm{H}, J=1.2,7.4$ $\left.\mathrm{Hz}, \mathrm{CH}_{2} \mathrm{Si}\right), 1.75\left(\mathrm{~s}, 3 \mathrm{H}, \mathrm{CH}_{2}=\mathrm{CCH}_{3}\right), 1.55$ (dt, 3H, $\left.J=1.3,6.2 \mathrm{~Hz}, \mathrm{CH}_{3} \mathrm{CH}=\mathrm{CH}\right), 1.21(\mathrm{~s}, 6 \mathrm{H}$, six of $\left.\left(\mathrm{CH}_{3}\right)_{2} \mathrm{CC}\left(\mathrm{CH}_{3}\right)_{2}\right), 1.12$ (s, 6H, six of $\left.\left(\mathrm{CH}_{3}\right)_{2} \mathrm{CC}\left(\mathrm{CH}_{3}\right)_{2}\right) ;{ }^{13} \mathrm{C} \mathrm{NMR}\left(100 \mathrm{MHz}, \mathrm{C}_{6} \mathrm{D}_{6}\right) \delta$ 154.3, 126.2, 124.0, 93.9, 81.8, 26.2, 26.1, 22.5, 18.4, 17.6; ${ }^{29} \mathrm{Si} \mathrm{NMR} \mathrm{(59} \mathrm{MHz,} \mathrm{CDCl}_{3}$ ) $\delta$-33.3; IR (film) 3114, 3020, 2980, 2936, 2887, 2858, 1645, 1445, 1390, 1370, 1271, 1182, 1162, 1146, 1063, 1007, 972, 933, 903, 879, 836, $817,759,642 \mathrm{~cm}^{-1}$.

Methallylenolsilane 8: ${ }^{1} \mathrm{H}$ NMR $\left(400 \mathrm{MHz}, \mathrm{C}_{6} \mathrm{D}_{6}\right) \delta 4.82\left(\mathrm{~m}, 1 \mathrm{H}\right.$, one of $\left.\mathrm{CH}_{2}=\mathrm{CCH}_{2}\right), 4.79(\mathrm{~m}, 1 \mathrm{H}$, one of $\left.\mathrm{CH}_{2}=\mathrm{CCH}_{2}\right), 4.59\left(\mathrm{~s}, 1 \mathrm{H}\right.$, one of $\left.\mathrm{CH}_{2}=\mathrm{CO}\right), 4.15\left(\mathrm{~s}, 1 \mathrm{H}\right.$, one of $\left.\mathrm{CH}_{2}=\mathrm{CO}\right), 1.86(\mathrm{~d}, 2 \mathrm{H}, J=0.8 \mathrm{~Hz}$, $\left.\mathrm{CH}_{2} \mathrm{Si}\right), 1.83$ (s, 3H, CH $\left.3 \mathrm{CO}\right), 1.75\left(\mathrm{~m}, 3 \mathrm{H}, \mathrm{CH}_{3} \mathrm{CCH}_{2}\right), 1.19\left(\mathrm{~s}, 6 \mathrm{H}\right.$, six of $\left.\left(\mathrm{CH}_{3}\right)_{2} \mathrm{CC}\left(\mathrm{CH}_{3}\right)_{2}\right), 1.11(\mathrm{~s}, 6 \mathrm{H}$, six of $\left.\left(\mathrm{CH}_{3}\right)_{2} \mathrm{CC}\left(\mathrm{CH}_{3}\right)_{2}\right) ;{ }^{13} \mathrm{C}$ NMR $\left(100 \mathrm{MHz}, \mathrm{C}_{6} \mathrm{D}_{6}\right) \delta 154.3,140.2,111.6,93.9,81.8,26.1$ (four Me), 25.2, 23.6, 22.5; ${ }^{29} \mathrm{Si} \mathrm{NMR}\left(59 \mathrm{MHz}, \mathrm{CDCl}_{3}\right.$ ) $\delta$-33.7; IR (film) 3078, 2980, 2936, 1646, 1446, 1390, $1371,1269,1147,1064,1008,972,933,880,842,814 \mathrm{~cm}^{-1}$.

(Z)-2-methylbut-2-enyl-enolsilane 10: ${ }^{1} \mathrm{H}$ NMR (400 MHz, $\left.\mathrm{C}_{6} \mathrm{D}_{6}\right) \delta 5.22(\mathrm{qm}, 1 \mathrm{H}, J=6.8 \mathrm{~Hz}$, $\left.\mathrm{CH}_{3} \mathrm{CH}=\mathrm{CCH}_{2}\right), 4.60\left(\mathrm{~s}, 1 \mathrm{H}\right.$, one of $\left.\mathrm{CH}_{2}=\mathrm{CO}\right), 4.15\left(\mathrm{~s}, 1 \mathrm{H}\right.$, one of $\left.\mathrm{CH}_{2}=\mathrm{CO}\right), 1.84-1.86(\mathrm{~m}, 5 \mathrm{H}$, $\left.\mathrm{CH}_{3} \mathrm{CCH}_{2} \mathrm{Si}\right), 1.75\left(\mathrm{~s}, 3 \mathrm{H}, \mathrm{CH}_{3} \mathrm{CO}\right), 1.63\left(\mathrm{dm}, 3 \mathrm{H}, J=6.8 \mathrm{~Hz}, \mathrm{CH}_{3} \mathrm{CH}=\mathrm{CCH}_{2}\right), 1.20(\mathrm{~s}, 6 \mathrm{H}$, six of $\left.\left(\mathrm{CH}_{3}\right)_{2} \mathrm{C}-\mathrm{C}\left(\mathrm{CH}_{3}\right)_{2}\right), 1.12$ (s, 6H, six of $\left.\left(\mathrm{CH}_{3}\right)_{2} \mathrm{CC}\left(\mathrm{CH}_{3}\right)_{2}\right) ;{ }^{13} \mathrm{C}$ NMR $\left(100 \mathrm{MHz}, \mathrm{C}_{6} \mathrm{D}_{6}\right) \delta 154.3,130.4$, 119.0, 93.7, 81.6, 26.3, 26.1, 26.0, 25.2, 23.6, 22.5, 17.7, 14.3; ${ }^{29} \mathrm{Si} \mathrm{NMR}\left(59 \mathrm{MHz}, \mathrm{CDCl}_{3}\right) \delta-33.3 ; \mathrm{IR}$ (film) 3115, 2980, 2931, 1647, 1445, 1373, 1271, 1146, 1062, 1007, 972, 931, 880, 821, 712, $643 \mathrm{~cm}^{-1}$. 


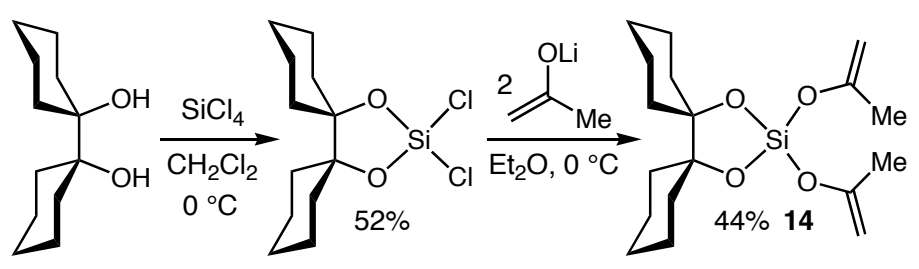

Preparation of dienolsilane 14: To a cooled $\left(0^{\circ} \mathrm{C}\right)$ solution of silicon tetrachloride $(17.3 \mathrm{~mL} ; 151$ $\mathrm{mmol})$ in $\mathrm{CH}_{2} \mathrm{Cl}_{2}(200 \mathrm{~mL})$ was added 1,8-diazabicyclo[5.4.0]undec-7-ene (15.7 $\left.\mathrm{mL} ; 105 \mathrm{mmol}\right)$. A solution of diol $1(10.0 \mathrm{~g} ; 50 \mathrm{mmol})$ in $\mathrm{CH}_{2} \mathrm{Cl}_{2}(100 \mathrm{~mL})$ was then added at room temperature with a water bath and the resultant solution was stirred for 12 hours. The solution was concentrated and the residue was treated with diethylether $(150 \mathrm{~mL})$. The mixture was stirred for 2 hour and then pentane $(50 \mathrm{~mL})$ was added. The mixture was then filtered and the filtrate was concentrated. The residue was dissolved in pentane $(100 \mathrm{~mL})$ then was filtered again. The filtrate was concentrated again to give $7.7 \mathrm{~g}$ $(52 \%)$ of 2 as a slightly yellow solid.

To a cooled $\left(0^{\circ} \mathrm{C}\right)$ solution of $\mathrm{MeLi}(24.6 \mathrm{~mL} ; 39 \mathrm{mmol} ; 1.6 \mathrm{M}$ in diethylether) was added 2(trimethylsilyloxy)propene $(5.1 \mathrm{~g} ; 39 \mathrm{mmol})$. The solution was stirred 30 minutes at $0^{\circ} \mathrm{C}$, and then warmed to room temperature and was stirred for 1 hour during which time the formation of a white precipitate was observed. The suspension was cooled to $0^{\circ} \mathrm{C}$, and the solution of $2(5.8 \mathrm{~g} ; 19.6 \mathrm{mmol})$ in diethylether $(30 \mathrm{~mL})$ was added. The solution was warmed to room temperature and stirred for 5 hours. The solution was diluted with pentane $(20 \mathrm{~mL})$, and the mixture was then filtered through a pad of celite. The filtrate was concentrated, and the residue was distilled in vacuo to give $2.9 \mathrm{~g}(44 \%)$ of 14 as a clear, colorless liquid. ${ }^{1} \mathrm{H}$ NMR (400 MHz, $\left.\mathrm{C}_{6} \mathrm{D}_{6}\right) \delta 4.67$ (s, 2H, two (one of $\left.\mathrm{C}=\mathrm{CH}_{2}\right)$ ), 4.18 (s, 2H, two (one of $\left.\mathrm{C}=\mathrm{CH}_{2}\right)$ ), 1.95-1.47 (m, 14H, cyclohexyl protons), 1.78 (s, 6H, two $\left.\mathrm{CH}_{3}\right)$, 1.06-0.92 (m, 6H, cyclohexyl protons); ${ }^{13} \mathrm{C}$ NMR (100 MHz, $\left.\mathrm{C}_{6} \mathrm{D}_{6}\right) \delta 153.9,94.3,83.6,33.1,26.4,22.8,22.1 ;{ }^{29} \mathrm{Si} \mathrm{NMR}(59 \mathrm{MHz}$, $\mathrm{CDCl}_{3}, \mathrm{SiMe}_{4}$ ) $\delta$-71.4; IR (thin film) 3115, 2936, 2861, 1649, 1450, 1373, 1274, 1255, 1070, $978 \mathrm{~cm}^{-1}$; LRMS (FAB+) calcd for $\mathrm{C}_{18} \mathrm{H}_{30} \mathrm{O}_{4} \mathrm{Si} 338$, found $(\mathrm{M}+\mathrm{H})^{+} 339$. 


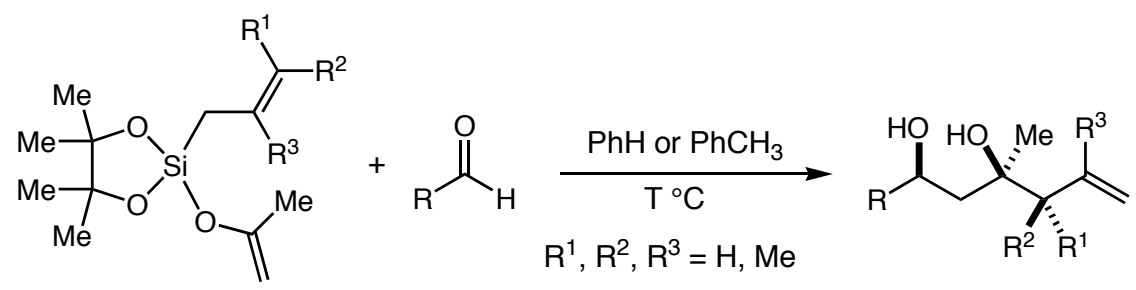

General procedure for the tandem aldol-allylation reactions: To a solution of the allylenolsilane $(0.63 \mathrm{mmol})$ in solvent (see Schemes 1 and 2 in the main text) $(0.4 \mathrm{~mL})$ was added cyclohexanecarboxaldehyde (48 $\mathrm{mg} ; 0.42 \mathrm{mmol}$ ). The solution was heated at the indicated temperature for the indicated time (see Schemes 1 and 2 in the main text). The solution was cooled and $1 \mathrm{M} \mathrm{HCl}$ (5 $\mathrm{mL}$ ) was added followed by ethyl acetate $(5 \mathrm{~mL})$. The mixture was stirred for $20 \mathrm{~min}$ and the layers were separated. The organic layer was washed with saturated aqueous $\mathrm{NaHCO}_{3}(5 \mathrm{~mL})$, washed with brine $(5$ $\mathrm{mL})$, dried $\left(\mathrm{Na}_{2} \mathrm{SO}_{4}\right)$, filtered, and concentrated. The residue was purified by chromatography on silica gel to give the diol products typically as a clear colorless oils.

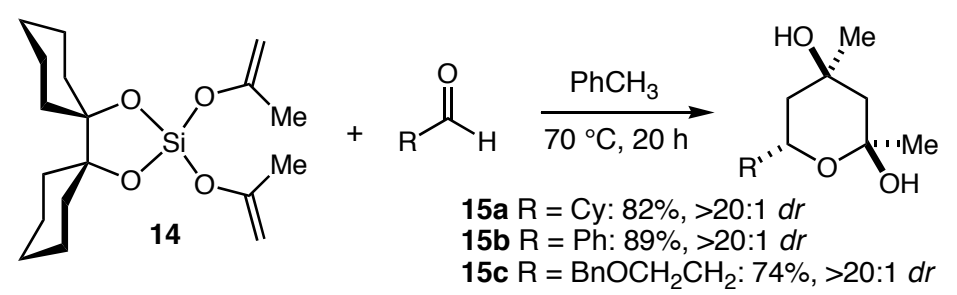

General procedure for the tandem aldol-aldol reactions: To a solution of dienolsilane $14(0.42$ mmol $)$ in toluene $(0.3 \mathrm{~mL})$ was added the aldehyde $(0.35 \mathrm{mmol})$. The solution was heated at $70{ }^{\circ} \mathrm{C}$ for 20 h. The solution was cooled and saturated aqueous sodium bicarbonate $(10 \mathrm{~mL})$ was added followed by ethyl acetate $(10 \mathrm{~mL})$. The mixture was stirred for $20 \mathrm{~min}$. Then the layers were separated and the aqueous layer was extracted with ethyl acetate $(10 \mathrm{~mL})$. The combined organic layers were washed with brine $(10 \mathrm{~mL})$, dried $\left(\mathrm{Na}_{2} \mathrm{SO}_{4}\right)$, filtered, and concentrated. The residue was purified by chromatography on silica gel to give the products as clear colorless oils, which solidified upon standing. 


\section{Characterization data for the tandem reaction products:}

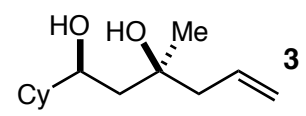

${ }^{1} \mathrm{H}$ NMR $\left(400 \mathrm{MHz}, \mathrm{CDCl}_{3}\right) \delta$ 5.82-5.93 (m, 1H, $\left.\mathrm{CH}=\mathrm{CH}_{2}\right)$, 5.08-5.17 (m, 2H, CH=CH$), 3.78$ (ddd, $\left.1 \mathrm{H}, J=1.9,5.6,10.7 \mathrm{~Hz}, \mathrm{c}-\mathrm{C}_{6} \mathrm{H}_{11} \mathrm{CHOH}\right), 3.11$ (br s, $2 \mathrm{H}$, two OH), 2.25 (d, $2 \mathrm{H}, J=7.4 \mathrm{~Hz}$, $\left.\mathrm{CH}_{2} \mathrm{CH}=\mathrm{CH}_{2}\right), 1.72-1.85\left(\mathrm{~m}, 3 \mathrm{H}\right.$, three of $\left.\mathrm{c}-\mathrm{C}_{6} \mathbf{H}_{11}\right), 1.57-1.71\left(\mathrm{~m}, 3 \mathrm{H}\right.$, one of $\mathrm{c}_{-} \mathrm{C}_{6} \mathrm{H}_{11} \mathrm{CHCH}_{2}$ and two of $\left.\mathrm{c}-\mathrm{C}_{6} \mathbf{H}_{11}\right), 1.47-1.52\left(\mathrm{dd}, 1 \mathrm{H}, J=1.9,14.5 \mathrm{~Hz}\right.$, one of $\left.\mathrm{c}^{-} \mathrm{C}_{6} \mathrm{H}_{11} \mathrm{CHCH}_{2}\right), 1.26\left(\mathrm{~s}, 3 \mathrm{H}, \mathrm{CH}_{3}\right), 0.95-1.36(\mathrm{~m}$, $6 \mathrm{H}$, six of $\left.\mathrm{c}-\mathrm{C}_{6} \mathbf{H}_{11}\right) ;{ }^{13} \mathrm{C}$ NMR $\left(100 \mathrm{MHz}, \mathrm{CDCl}_{3}\right) \delta 133.6,118.9,73.5,73.0,49.1,44.3,42.9,28.8,28.0$, 26.5, 26.3, 26.2, 25.6; IR (film) 3354, 3077, 2926, 2854, 1641, 1450, 1376, 1130, 1064, 998, 915, 880, $842 \mathrm{~cm}^{-1}$; LRMS (FAB+) calc'd for $\mathrm{C}_{13} \mathrm{H}_{24} \mathrm{O}_{2} 212$, found $(\mathrm{M}+\mathrm{H})^{+} 213$.

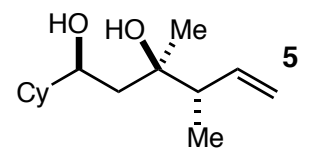

${ }^{1} \mathrm{H}$ NMR $\left(400 \mathrm{MHz}, \mathrm{CDCl}_{3}\right) \delta 5.75-5.85\left(\mathrm{ddd}, 1 \mathrm{H}, J=8.6,11.1,16.3 \mathrm{~Hz}, \mathrm{CH}=\mathrm{CH}_{2}\right), 5.12(\mathrm{~m}, 1 \mathrm{H}$, one of $\left.\mathrm{CH}=\mathrm{CH}_{2}\right), 5.07-5.10\left(\mathrm{~m}, 1 \mathrm{H}\right.$, one of $\left.\mathrm{CH}=\mathrm{CH}_{2}\right), 3.76\left(\mathrm{ddd}, 1 \mathrm{H}, J=2.6,5.6,9.7 \mathrm{~Hz}, \mathrm{c}-\mathrm{C}_{6} \mathrm{H}_{11} \mathrm{CHOH}\right)$, 3.27 (br s, 2H, two OH), 2.17-2.25 (m, 1H, $\left.\mathrm{CHCH}_{3}\right), 1.63-1.86\left(\mathrm{~m}, 5 \mathrm{H}\right.$, five of c- $\left.\mathrm{C}_{6} \mathbf{H}_{11}\right), 1.48-1.61(\mathrm{~m}$, $\left.2 \mathrm{H}, \mathrm{c}-\mathrm{C}_{6} \mathrm{H}_{11} \mathrm{CHCH}_{2}\right), 1.20\left(\mathrm{~s}, 3 \mathrm{H}, \mathrm{CH}_{3} \mathrm{COH}\right), 1.12-1.37\left(\mathrm{~m}, 4 \mathrm{H}\right.$, four of $\left.\mathrm{c}-\mathrm{C}_{6} \mathbf{H}_{11}\right), 1.03(\mathrm{~d}, 3 \mathrm{H}, J=6.9 \mathrm{~Hz}$, $\left.\mathrm{CHCH}_{3}\right), 0.95-1.11\left(\mathrm{~m}, 2 \mathrm{H}\right.$, two of $\left.\mathrm{c}-\mathrm{C}_{6} \mathbf{H}_{11}\right) ;{ }^{13} \mathrm{C} \mathrm{NMR}\left(100 \mathrm{MHz}, \mathrm{CDCl}_{3}\right) \delta 139.6,116.5,74.8,73.1$, 50.3, 44.4, 40.6, 29.0, 28.2, 26.7, 26.4, 26.3, 23.1, 14.9; IR (film) 3350, 3077, 2974, 2924, 2853, 1637 , 1450, 1420, 1375, 1280, 1188, 1128, 1063, 1002, 939, 913, $838 \mathrm{~cm}^{-1}$; LRMS (FAB+) calc'd for $\mathrm{C}_{14} \mathrm{H}_{26} \mathrm{O}_{2}$ 226 , found $(\mathrm{M}+\mathrm{H})^{+} 227$.

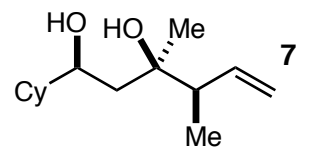

${ }^{1} \mathrm{H}$ NMR $\left(400 \mathrm{MHz}, \mathrm{CDCl}_{3}\right) \delta$ 5.76-5.86 $\left(\mathrm{m}, 1 \mathrm{H}, \mathrm{CH}=\mathrm{CH}_{2}\right), 5.12\left(\mathrm{~m}, 1 \mathrm{H}\right.$, one of $\left.\mathrm{CH}=\mathrm{CH}_{2}\right)$, 5.07-5.10 $\left(\mathrm{m}, 1 \mathrm{H}\right.$, one of $\left.\mathrm{CH}=\mathrm{CH}_{2}\right), 3.76\left(\mathrm{~m}, 1 \mathrm{H}, \mathrm{c}-\mathrm{C}_{6} \mathrm{H}_{11} \mathrm{CHOH}\right), 3.31$ (br s, 1H, OH), 2.80 (br s, 1H, OH), 2.18$2.26\left(\mathrm{~m}, 1 \mathrm{H}, \mathrm{CHCH}_{3}\right), 1.72-1.87\left(\mathrm{~m}, 3 \mathrm{H}\right.$, three of c- $\left.\mathrm{C}_{6} \mathbf{H}_{11}\right), 1.63-1.70\left(\mathrm{~m}, 2 \mathrm{H}\right.$, two of c- $\left.\mathrm{C}_{6} \mathbf{H}_{11}\right), 1.46-1.57$ 
(m, $\left.2 \mathrm{H}, \mathrm{c}-\mathrm{C}_{6} \mathrm{H}_{11} \mathrm{CHCH}_{2}\right), 1.24$ (s, 3H, $\left.\mathrm{CH}_{3} \mathrm{COH}\right), 1.11-1.35$ (m, 4H, four of $\left.\mathrm{c}-\mathrm{C}_{6} \mathbf{H}_{11}\right), 1.04$ (d, 3H, J=6.9 $\left.\mathrm{Hz}, \mathrm{CHCH}_{3}\right), 0.95-1.10\left(\mathrm{~m}, 2 \mathrm{H}\right.$, two of $\left.\mathrm{c}-\mathrm{C}_{6} \mathbf{H}_{11}\right) ;{ }^{13} \mathrm{C} \mathrm{NMR}\left(100 \mathrm{MHz}, \mathrm{CDCl}_{3}\right) \delta$ 139.9, 116.2, 74.9, 73.6, 50.4, 44.4, 40.1, 29.0, 28.2, 26.7, 26.4, 26.3, 23.2, 14.6; IR (film) 3350, 3077, 2974, 2924, 2852, 1637, 1450, 1419, 1378, 1309, 1102, 1062, 1038, 998, 912, $835 \mathrm{~cm}^{-1}$; LRMS (FAB+) calc'd for $\mathrm{C}_{14} \mathrm{H}_{26} \mathrm{O}_{2} 226$, found $(\mathrm{M}+\mathrm{H})^{+} 227$.

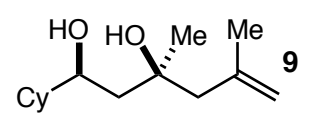

${ }^{1} \mathrm{H}$ NMR $\left(400 \mathrm{MHz}, \mathrm{CDCl}_{3}\right) \delta 4.94\left(\mathrm{~m}, 1 \mathrm{H}\right.$, one of $\left.\mathrm{CH}_{2} \mathrm{C}=\mathrm{CH}_{2}\right), 4.75\left(\mathrm{~m}, 1 \mathrm{H}\right.$, one of $\left.\mathrm{CH}_{2} \mathrm{C}=\mathrm{CH}_{2}\right)$,

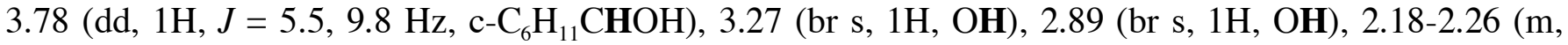
$\left.2 \mathrm{H}, \mathrm{CH}_{2} \mathrm{C}=\mathrm{CH}_{2}\right), 1.85\left(\mathrm{~s}, 3 \mathrm{H}, \mathrm{CH}_{3} \mathrm{C}=\mathrm{CH}_{2}\right), 1.72-1.86\left(\mathrm{~m}, 3 \mathrm{H}\right.$, three of $\left.\mathrm{c}-\mathrm{C}_{6} \mathbf{H}_{11}\right), 1.60-1.71(\mathrm{~m}, 3 \mathrm{H}$, one of c- $\mathrm{C}_{6} \mathrm{H}_{11} \mathrm{CHCH}_{2}$ and two of $\left.\mathrm{c}-\mathrm{C}_{6} \mathbf{H}_{11}\right), 1.47-1.52\left(\mathrm{dd}, 1 \mathrm{H}, J=1.8,14.5 \mathrm{~Hz}\right.$, one of c- $\left.\mathrm{C}_{6} \mathrm{H}_{11} \mathrm{CHCH}_{2}\right), 1.28(\mathrm{~s}$, $\left.3 \mathrm{H}, \mathrm{CH}_{3} \mathrm{COH}\right), 1.11-1.36\left(\mathrm{~m}, 4 \mathrm{H}\right.$, four of $\left.\mathrm{c}^{-} \mathrm{C}_{6} \mathbf{H}_{11}\right), 0.95-1.10\left(\mathrm{~m}, 2 \mathrm{H}\right.$, two of $\left.\mathrm{c}^{-} \mathrm{C}_{6} \mathbf{H}_{11}\right) ;{ }^{13} \mathrm{C}$ NMR (100 $\left.\mathrm{MHz}, \mathrm{CDCl}_{3}\right) \delta 142.1,115.0,73.4,73.2,52.2,44.4,43.3,29.0,28.3,26.7,26.4,26.3,26.2,25.2$; IR (film) 3350, 3073, 2924, 2853, 1643, 1450, 1376, 1318, 1260, 1186, 1130, 1039, 981, 892, 860, $735 \mathrm{~cm}^{-1}$; LRMS (FAB+) calc'd for $\mathrm{C}_{14} \mathrm{H}_{26} \mathrm{O}_{2} 226$, found $(\mathrm{M}+\mathrm{H})^{+} 227$.

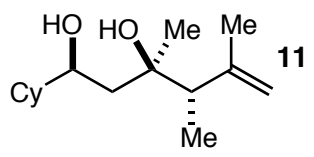

${ }^{1} \mathrm{H}$ NMR $\left(400 \mathrm{MHz}, \mathrm{CDCl}_{3}\right) \delta 4.91\left(\mathrm{~m}, 1 \mathrm{H}\right.$, one of $\left.\mathrm{CH}_{3} \mathrm{C}=\mathrm{CH}_{2}\right), 4.78\left(\mathrm{~m}, 1 \mathrm{H}\right.$, one of $\left.\mathrm{CH}_{3} \mathrm{C}=\mathrm{CH}_{2}\right)$, 3.73-3.78 (m, 1H, c- $\mathrm{C}_{6} \mathrm{H}_{11} \mathrm{CHOH}$ ), 3.46 (br s, 1H, OH), 2.75 (br s, 1H, OH), 2.25 (q, 1H, J = 7.2 Hz, $\left.\mathrm{CHCH}_{3}\right), 1.79\left(\mathrm{~s}, 3 \mathrm{H}, \mathrm{CH}_{3} \mathrm{C}=\mathrm{CH}_{2}\right), 1.63-1.85\left(\mathrm{~m}, 5 \mathrm{H}\right.$, five of $\left.\mathrm{c}-\mathrm{C}_{6} \mathbf{H}_{11}\right), 1.50-1.62(\mathrm{~m}, 2 \mathrm{H}, \mathrm{c}-$ $\left.\mathrm{C}_{6} \mathrm{H}_{11} \mathrm{CHCH}_{2}\right), 1.22\left(\mathrm{~s}, 3 \mathrm{H}, \mathrm{CH}_{3} \mathrm{COH}\right), 1.11-1.38\left(\mathrm{~m}, 4 \mathrm{H}\right.$, four of $\left.\mathrm{c}^{-\mathrm{C}_{6}} \mathbf{H}_{11}\right), 1.08(\mathrm{~d}, 3 \mathrm{H}, J=7.2 \mathrm{~Hz}$, $\left.\mathrm{CHCH}_{3}\right), 0.95-1.10\left(\mathrm{~m}, 2 \mathrm{H}\right.$, two of $\left.\mathrm{c}^{-} \mathrm{C}_{6} \mathbf{H}_{11}\right) ;{ }^{13} \mathrm{C} \mathrm{NMR}\left(100 \mathrm{MHz}, \mathrm{CDCl}_{3}\right) \delta$ 147.2, 113.8, 74.9, 73.2, 52.4, 44.4, 41.8, 29.1, 28.3, 26.7, 26.5, 26.4, 23.6, 22.1, 14.4; IR (film) 3368, 3072, 2974, 2925, 2853, 1640, 1450, 1375, 1133, 1066, 981, 937, 892, 863, $841 \mathrm{~cm}^{-1}$; LRMS (FAB+) calc'd for $\mathrm{C}_{15} \mathrm{H}_{28} \mathrm{O}_{2} 240$, found $(\mathrm{M}+\mathrm{H})^{+} 241$. 


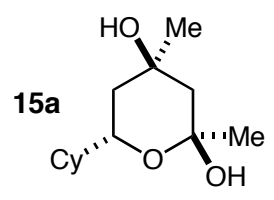

${ }^{1} \mathrm{H}$ NMR (400 MHz, $\left.\mathrm{C}_{6} \mathrm{D}_{6}\right) \delta 4.46(\mathrm{~s}, 1 \mathrm{H}, \mathrm{OH}), 3.89$ (ddd, $\left.J=11.8,6.3,2.3 \mathrm{~Hz}, 1 \mathrm{H}, \mathrm{OCH}\right), 3.35$ (s, 1H, OH), 2.10-1.00 (m, 11H, $\left.\mathrm{C}_{6} \mathbf{H}_{11}\right), 1.67\left(\mathrm{dd}, J=13.7,2.3 \mathrm{~Hz}, 1 \mathrm{H}\right.$, one of $\left.\mathrm{CH}_{2}\right), 1.46(\mathrm{dt}, J=13.2,2.3$ $\mathrm{Hz}, 1 \mathrm{H}$, one of $\left.\mathrm{CH}_{2}\right), 1.19\left(\mathrm{~d}, J=13.6 \mathrm{~Hz}, 1 \mathrm{H}\right.$, one of $\left.\mathrm{CH}_{2}\right), 1.09\left(\mathrm{dd}, J=13.2,12.0 \mathrm{~Hz}, 1 \mathrm{H}\right.$, one of $\left.\mathrm{CH}_{2}\right)$, 1.37 (s, 3H, CH 3$), 1.06$ (s, 3H, CH 3$) ;{ }^{13} \mathrm{C}$ NMR (100 MHz, $\left.\mathrm{C}_{6} \mathrm{D}_{6}\right) \delta$ 96.8, 70.0, 69.6, 45.9, 43.1, 40.9, 31.3, 30.1, 29.7, 29.4, 27.4, 27.1, 27.0; IR (thin film) 3338, 2924, 2852, 1451, 1375, 1230, 1194, 1036, 973, 908, 860, $824 \mathrm{~cm}^{-1}$; LRMS (FAB+) calcd for $\mathrm{C}_{13} \mathrm{H}_{24} \mathrm{O}_{3} 228$, found $(\mathrm{M}+\mathrm{H})^{+} 229$.

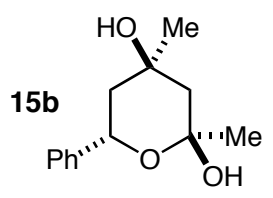

${ }^{1} \mathrm{H}$ NMR $\left(500 \mathrm{MHz}, \mathrm{CDCl}_{3}\right) \delta$ 7.42-7.26 (m, 5H, $\left.\mathrm{C}_{6} \mathbf{H}_{5}\right), 5.17(\mathrm{dd}, J=12.0,2.5 \mathrm{~Hz}, 1 \mathrm{H}, \mathrm{OCH}), 4.66(\mathrm{~s}$, 1H, OH), $3.86(\mathrm{~s}, 1 \mathrm{H}, \mathbf{O H}), 1.94\left(\mathrm{dd}, J=14.0,2.0 \mathrm{~Hz}, 1 \mathrm{H}\right.$, one of $\left.\mathrm{CH}_{2}\right), 1.89$ (dt, $J=14.0,2.5 \mathrm{~Hz}, 1 \mathrm{H}$, one of $\left.\mathrm{CH}_{2}\right), 1.65\left(\mathrm{~d}, J=14.0 \mathrm{~Hz}, 1 \mathrm{H}\right.$, one of $\left.\mathrm{CH}_{2}\right), 1.58\left(\mathrm{dd}, J=14.0,12.0 \mathrm{~Hz}, 1 \mathrm{H}\right.$, one of CH $\left.\mathbf{H}_{2}\right), 1.51(\mathrm{~s}$, $\left.3 \mathrm{H}, \mathrm{CH}_{3}\right), 1.28\left(\mathrm{~s}, 3 \mathrm{H}, \mathrm{CH}_{3}\right) ;{ }^{13} \mathrm{C}$ NMR (100 MHz, $\left.\mathrm{C}_{6} \mathrm{D}_{6}\right) \delta$ 142.0, 128.1, 127.1, 125.9, 97.4, 69.6, 68.5, 45.9, 44.9, 30.6, 29.8; IR (thin film) 3352, 2961, 2922, 1452, 1233, 1194, 1041, 1018, 910, $854 \mathrm{~cm}^{-1}$; LRMS (FAB+) calcd for $\mathrm{C}_{13} \mathrm{H}_{18} \mathrm{O}_{3} 222$, found $(\mathrm{M}-\mathrm{H})^{+} 221$.

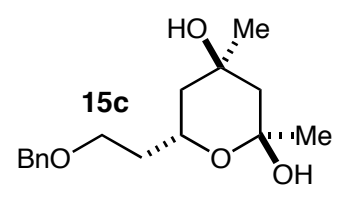

${ }^{1} \mathrm{H}$ NMR (400 MHz, $\left.\mathrm{C}_{6} \mathrm{D}_{6}\right) \delta$ 7.35-7.30 (m, 2H, two of $\left.\mathrm{C}_{6} \mathbf{H}_{5}\right), \delta$ 7.21-7.06 (m, 3H, three of $\left.\mathrm{C}_{6} \mathbf{H}_{5}\right)$, $4.37\left(\mathrm{~d}, J=12.2 \mathrm{~Hz}, 2 \mathrm{H}, \mathrm{OCH}_{2} \mathrm{Ph}\right), 4.34(\mathrm{~s}, 1 \mathrm{H}, \mathrm{OH}), 4.34-4.28(\mathrm{~m}, 1 \mathrm{H}, \mathrm{OCH}), 3.68-3.51(\mathrm{~m}, 2 \mathrm{H}$, $\left.\mathrm{BnOCH}_{2}\right), 2.71(\mathrm{~s}, 1 \mathrm{H}, \mathrm{OH}), 1.92-1.72\left(\mathrm{~m}, 2 \mathrm{H}, \mathrm{BnOCH}_{2} \mathrm{CH}_{2}\right), 1.61(\mathrm{dd}, J=13.7,2.3 \mathrm{~Hz}, 1 \mathrm{H}$, one of $\left.\mathrm{CH}_{2}\right), 1.35\left(\mathrm{~s}, 3 \mathrm{H}, \mathrm{CH}_{3}\right), 1.31\left(\mathrm{dt}, J=13.4,2.3 \mathrm{~Hz}, 1 \mathrm{H}\right.$, one of $\left.\mathrm{CH}_{2}\right), 1.14(\mathrm{~d}, J=13.7 \mathrm{~Hz}, 1 \mathrm{H}$, one of $\left.\mathrm{CH}_{2}\right), 0.99\left(\mathrm{dd}, J=13.4,11.8 \mathrm{~Hz}, 1 \mathrm{H}\right.$, one of $\left.\mathrm{CH}_{2}\right), 0.92\left(\mathrm{~s}, 3 \mathrm{H}, \mathrm{CH}_{3}\right) ;{ }^{13} \mathrm{C} \mathrm{NMR}\left(100 \mathrm{MHz}, \mathrm{C}_{6} \mathrm{D}_{6}\right) \delta$ 
$139.3,128.5,127.9,127.6,96.8,73.1,69.6,67.3,63.5,45.7,44.3,36.8,31.2,30.1$; IR (thin film) 3351 , $3233,3061,2950,2890,1448,1417,1376,1193,1104,1051,977,906,741 \mathrm{~cm}^{-1}$.

\section{Proof of relative stereochemistry for the tandem reaction products:}

Diol 3 was treated with $\mathrm{HgCl}(\mathrm{OAc})$ in $\mathrm{CH}_{2} \mathrm{Cl}_{2}$, giving a single product in $88 \%$ yield (Scheme 1). The minor product from the tandem reaction (3a) was subjected to the same conditions, resulting in an $81 \%$ yield of 2 compounds in a 1:1 ratio. The illustrated selective 1D NOESY enhancements on the product of the first reaction and on one of the products of the second reaction conclusively established that $\mathbf{3}$ posseses a syn diol relationship.

\section{Scheme 1}
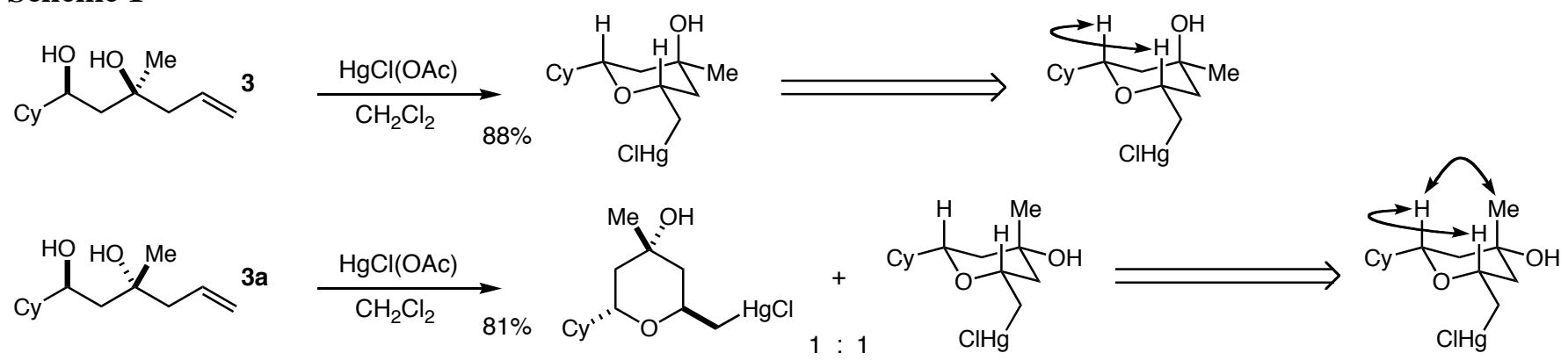

Diol 5 was treated with $\mathrm{HgCl}(\mathrm{OAc})$ in $\mathrm{CH}_{2} \mathrm{Cl}_{2}$, giving a single product in $62 \%$ yield (Scheme 2). The illustrated selective 1D NOESY enhancement, combined with the illustrated coupling constant conclusively established the relative stereochemistry of $\mathbf{5}$ as shown.

\section{Scheme 2}

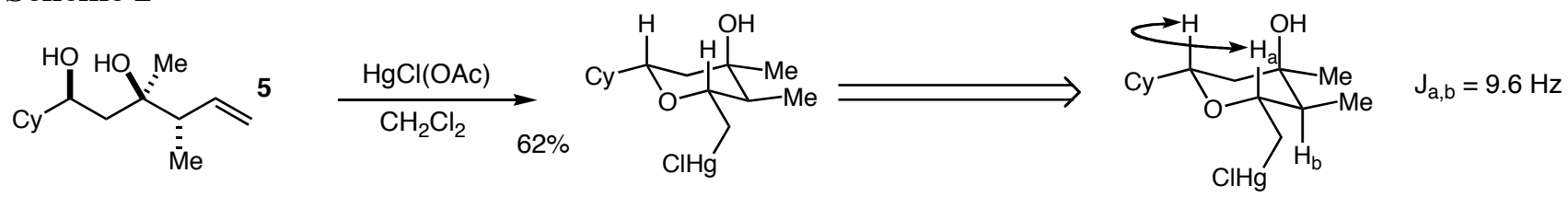


Diol 7 was treated with $\mathrm{HgCl}(\mathrm{OAc})$ in $\mathrm{CH}_{2} \mathrm{Cl}_{2}$, giving a single product in $89 \%$ yield (Scheme 3). The illustrated selective 1D NOESY enhancement, combined with the illustrated coupling constant conclusively established the relative stereochemistry of $\mathbf{7}$ as shown.

\section{Scheme 3}

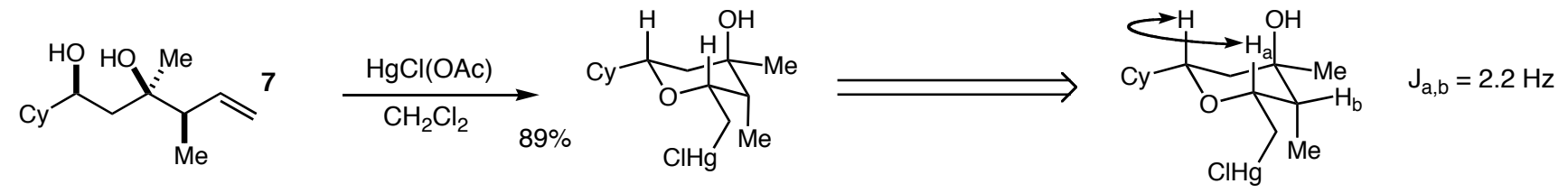

The relative stereochemistry of diol $\mathbf{9}$ was assigned by analogy to that of diol $\mathbf{3}$, which was proven conclusively (see above).

Diol 11 was treated with $\mathrm{HgCl}(\mathrm{OAc})$ in $\mathrm{CH}_{2} \mathrm{Cl}_{2}$, giving a mixture of products from which the illustrated tetrahydropyran was isolated in 35\% yield (Scheme 4). The illustrated selective 1D NOESY enhancements established the relative stereochemistry of $\mathbf{1 1}$ as shown.

\section{Scheme 4}<smiles>[Y10]C(=C)C([14CH3])C(O)(O)CC(O)C1CCCCC1</smiles>
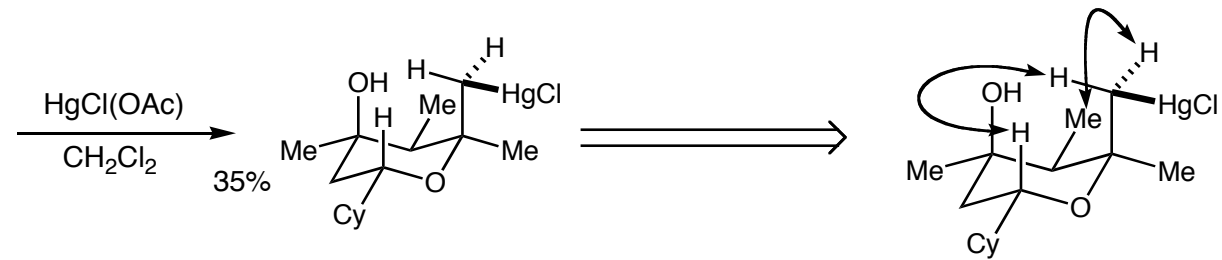

The relative stereochemistry of Hemiketals $\mathbf{1 5 a}, \mathbf{1 5 b}$, and $\mathbf{1 5 c}$ was established by way of the illustrated selective 1D NOESY enhancements (Scheme 5).

\section{Scheme 5}
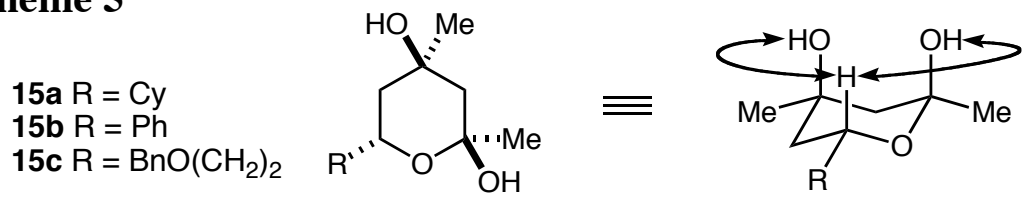
${ }^{1}$ H NMR spectra of the tandem aldol-allylation and tandem aldol-aldol products:

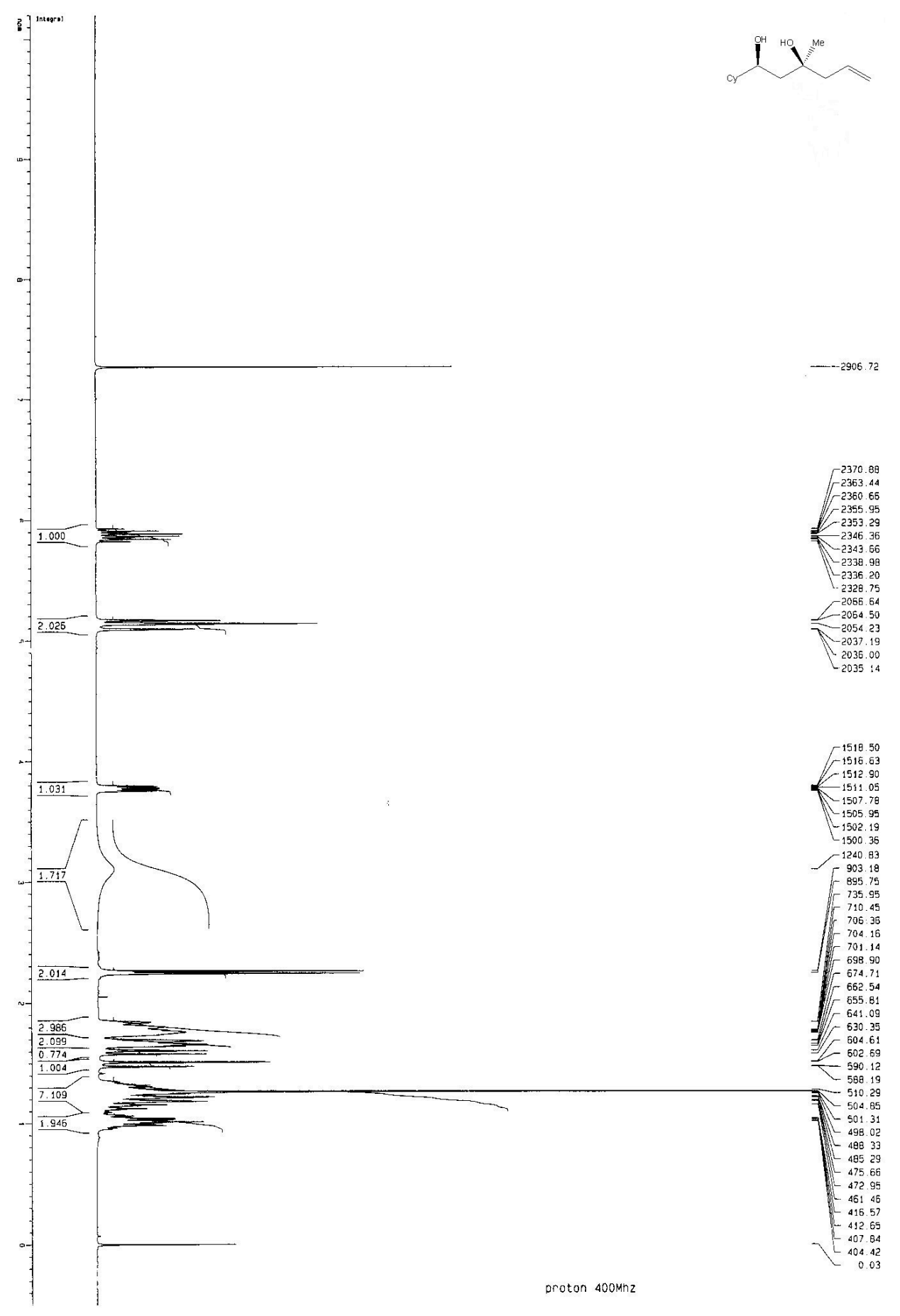


Page S12

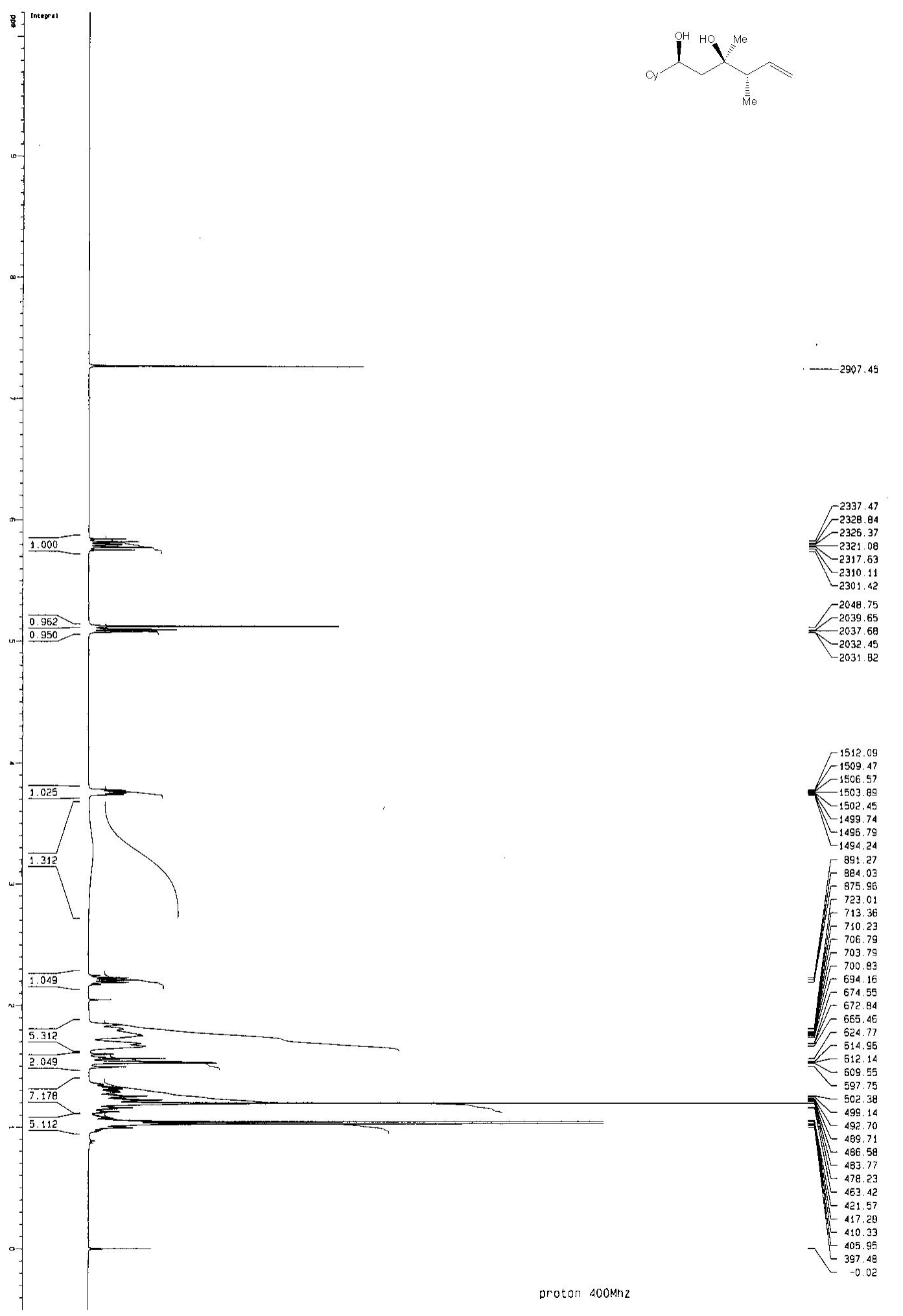


Page S13

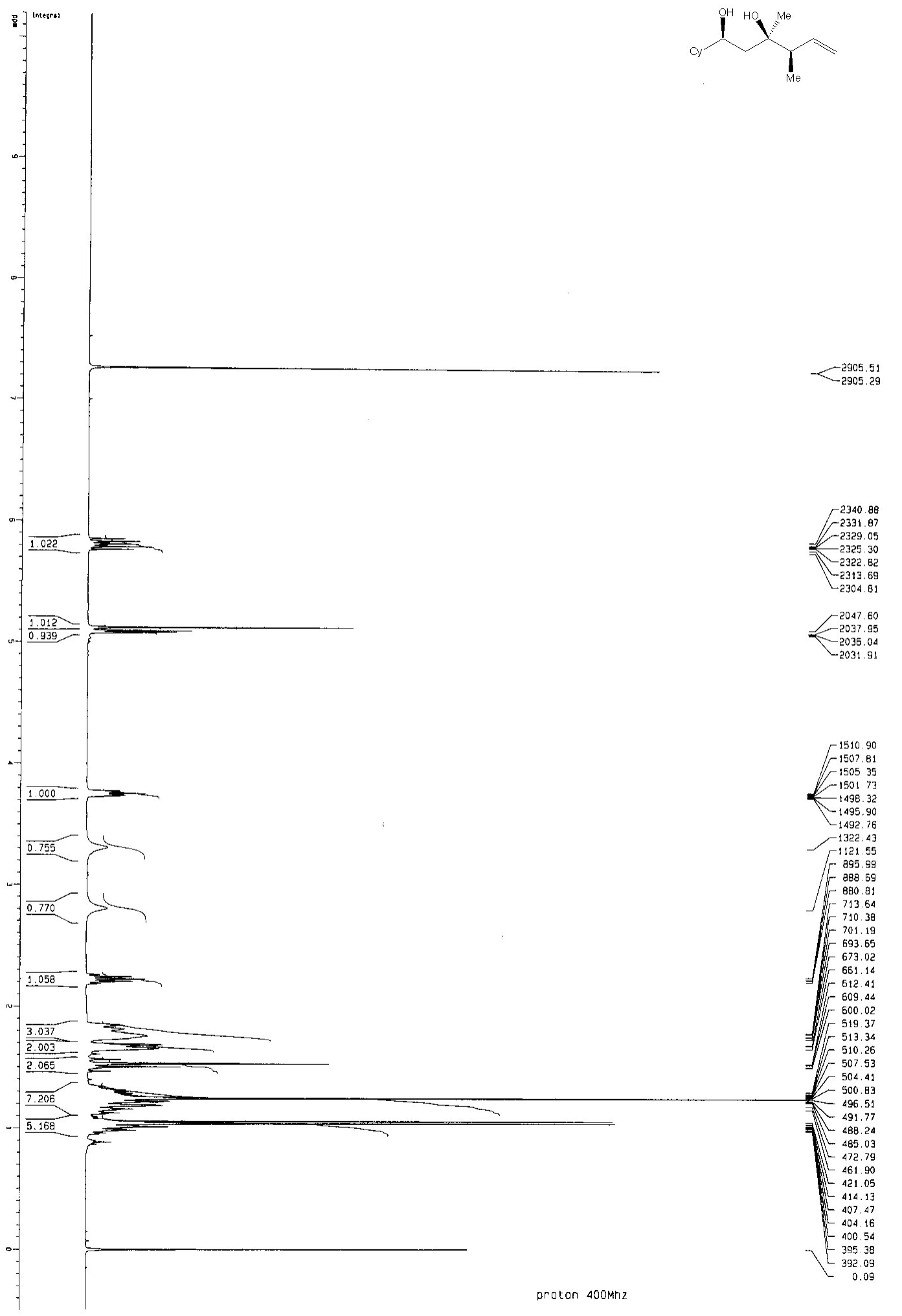


Page S14

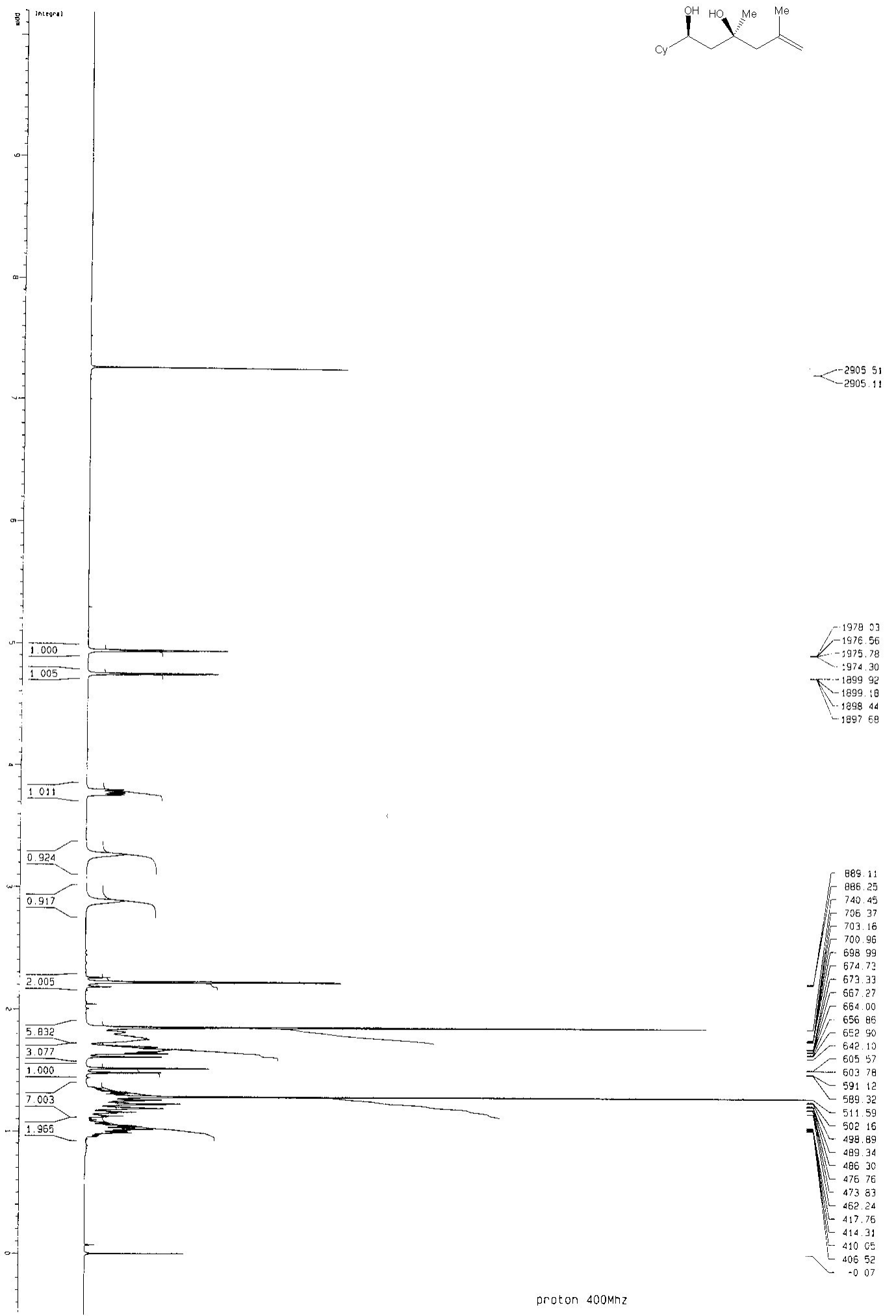


Page S15

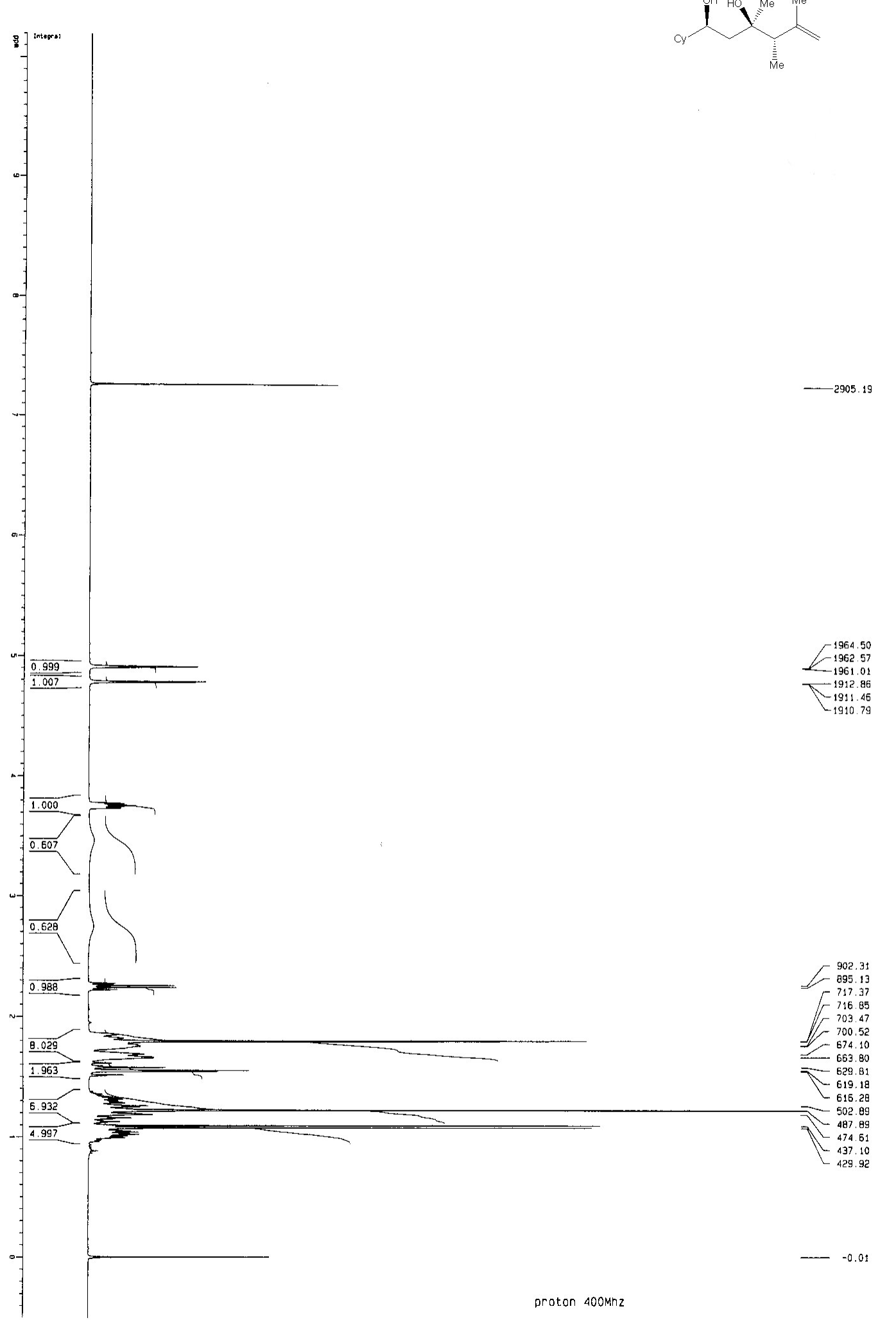


Page S16
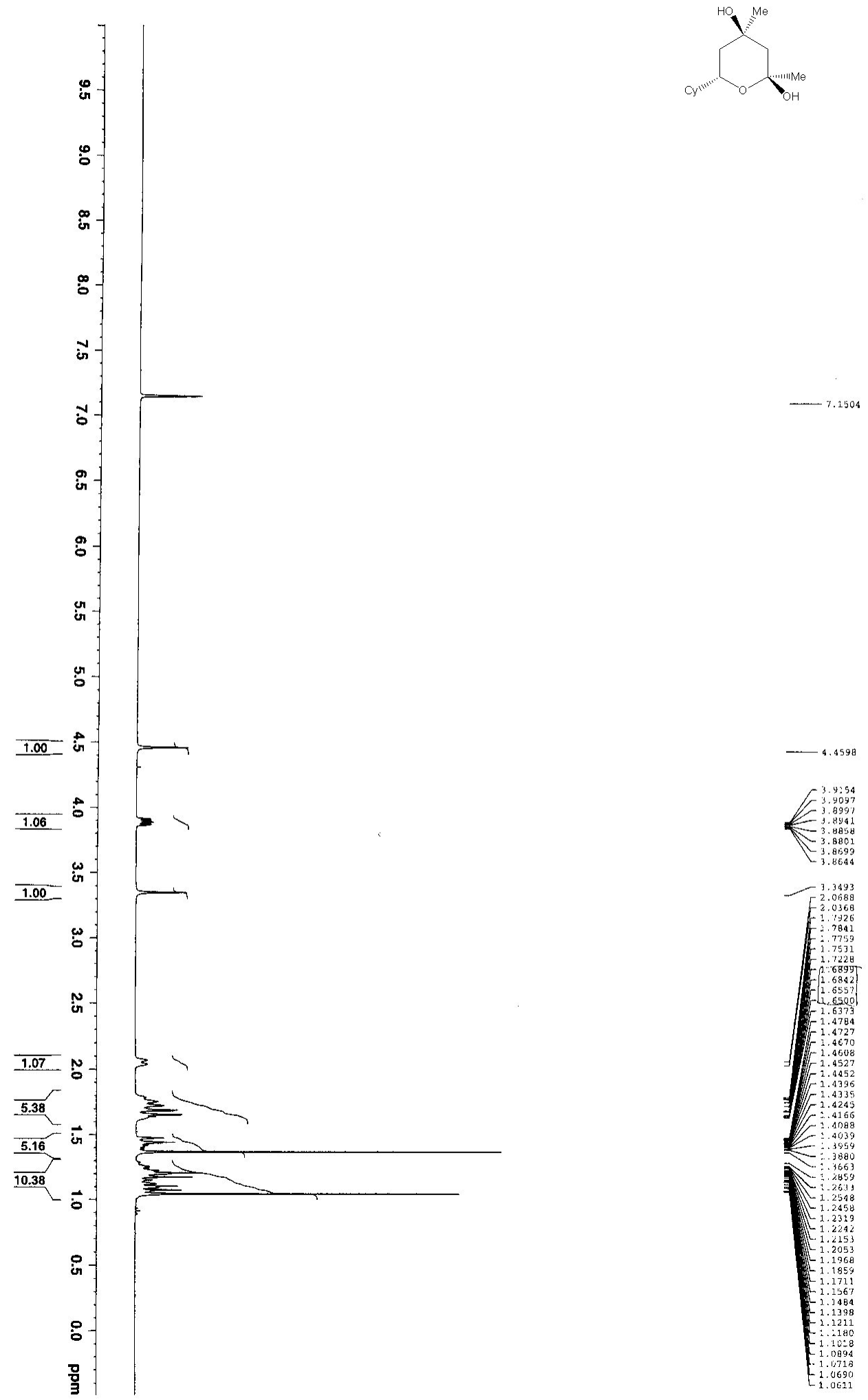
Page S17
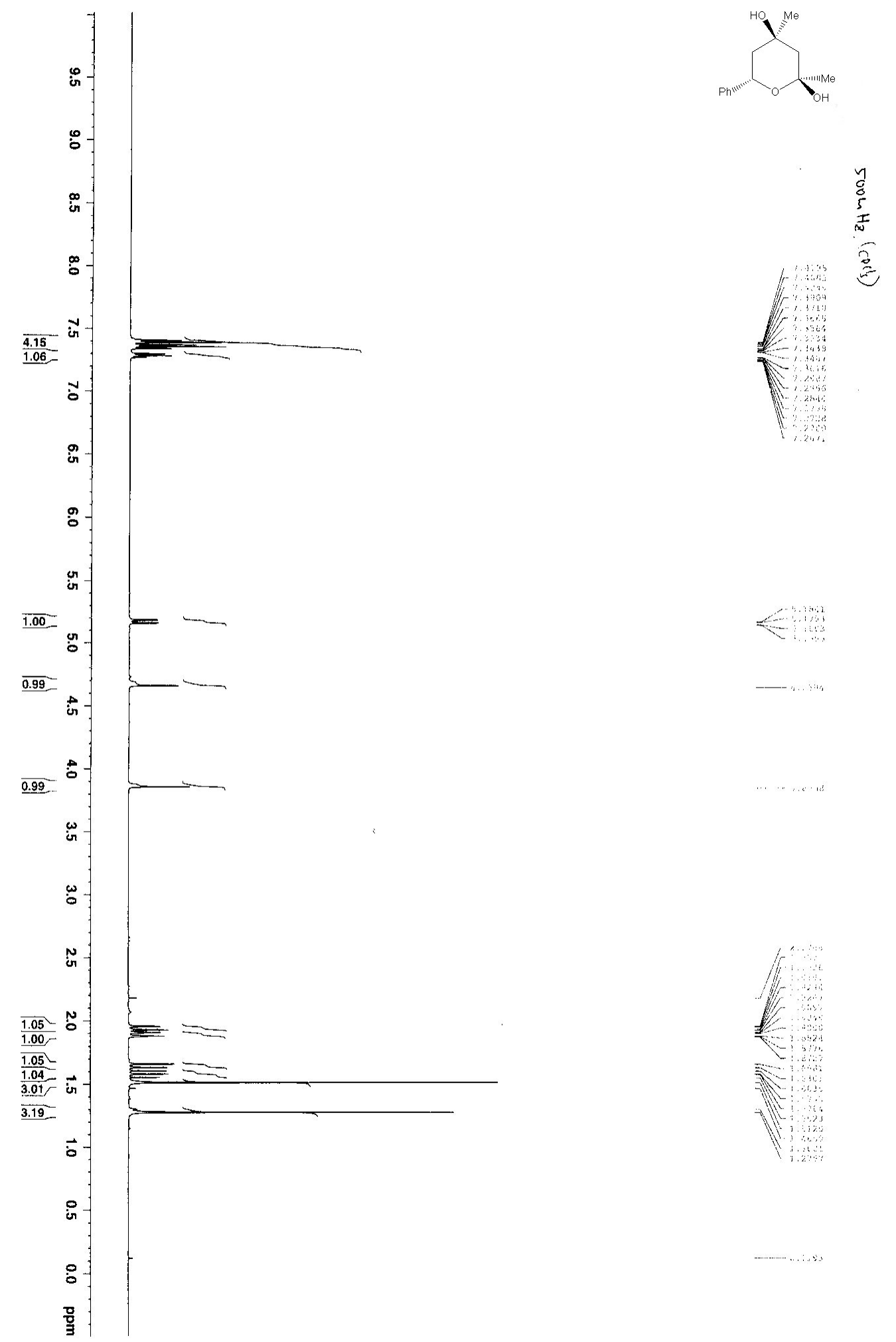
Page S18
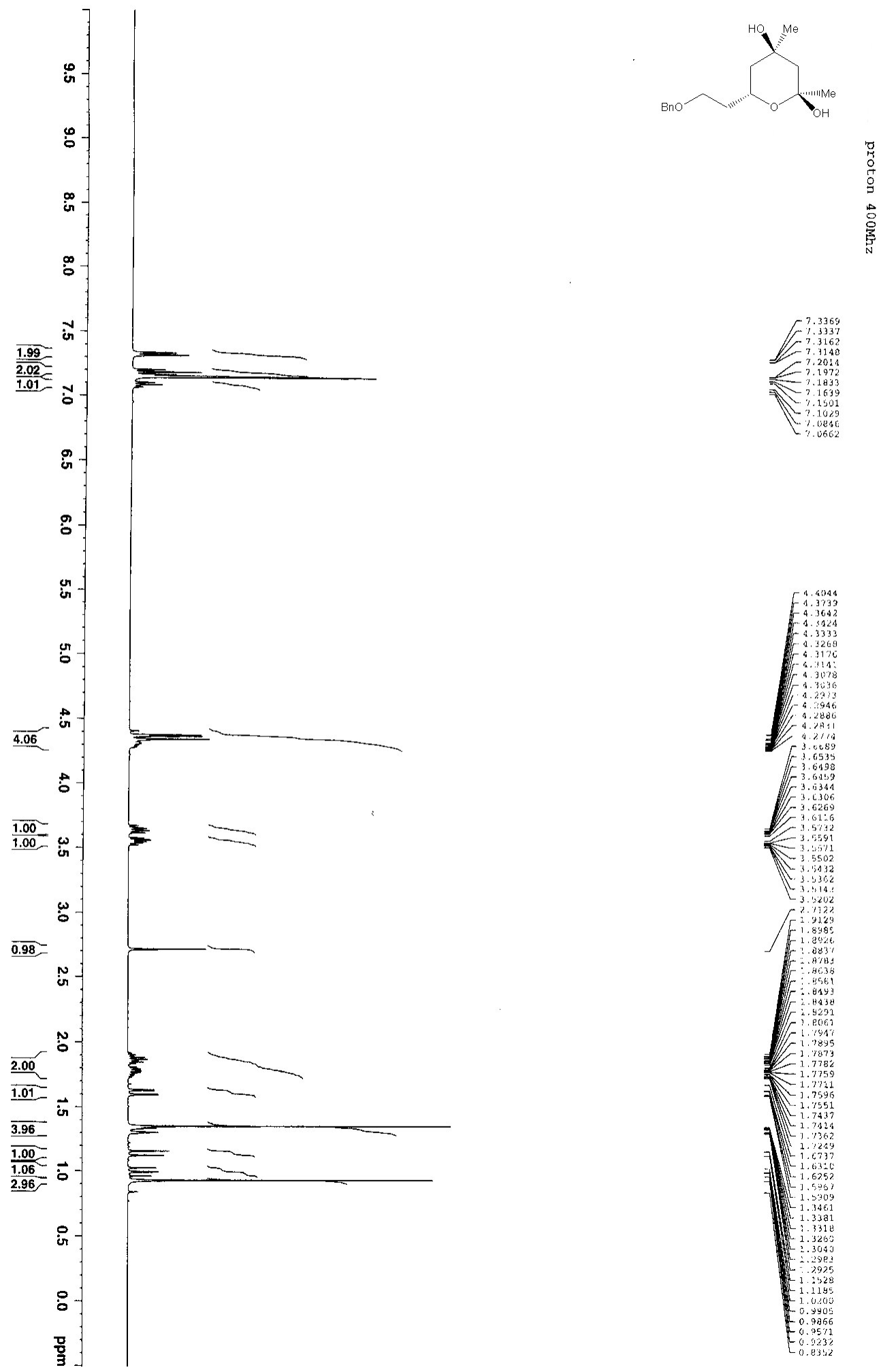

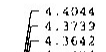

8. 4.3333

C. 4.40

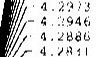

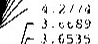

for.6.6939

F

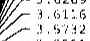

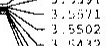

(2.,

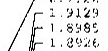

Fent

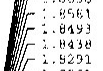

- 1.7873

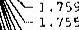

- 1.81824

W 1.8362

W 1.6252

Atr $\begin{array}{r}1.3331 \\ -3318\end{array}$

1.3260
-.3049
-2.045

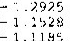

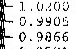

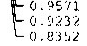

